\title{
S-adenosyl methionine regulates calcium channels and inhibits uterine smooth muscle contraction in rats with infectious premature delivery through the transient receptor protein 3/protein kinase $C \beta / C$-kinase-activated protein phosphatase-1 inhibitor of $17 \mathrm{kDa}$ signaling pathway
}

\author{
JING GE $^{1 *}$, TAO HAN ${ }^{2 *}$, XIAOQIU LI ${ }^{3 *}$, LILI SHAN $^{1}$, JINHUAN ZHANG $^{1}$, YAN HONG ${ }^{1}$, \\ YANQIU XIA $^{1}$, JUN WANG ${ }^{1}$ and MINGXIAO HOU ${ }^{4}$ \\ Departments of ${ }^{1}$ Maternity, ${ }^{2}$ Oncology, ${ }^{3}$ Neurology and ${ }^{4}$ Cardiothoracic Surgery, \\ General Hospital of Shenyang Military Area Command, Shenyang, Liaoning 110016, P.R. China
}

Received November 10, 2017; Accepted March 9, 2018

DOI: $10.3892 /$ etm.2018.6164

\begin{abstract}
The aim of the present study was to investigate the effects of S-adenosyl methionine (SAMe) on infectious premature inflammatory factors and uterine contraction, and to further explore its mechanism of action via the transient receptor protein 3 (TRPC3)/protein kinase $\mathrm{C} \beta$ (PKC $\beta$ )/C-kinase-activated protein phosphatase-1 inhibitor of $17 \mathrm{kDa}$ (CPI-17) signaling pathway, following intervention by a TRPC3 inhibitor. A rat model of premature delivery induced by lipopolysaccharide (LPS) was established. Following treatment with SAMe and inhibiting TRPC3 expression, rat serum and uterus were isolated. Hematoxylin and eosin staining was used to observe the histopathological changes in the uterus. Uterine muscle strips in vitro were selected to measure the changes in muscle tension. ELISA was utilized to measure the changes in serum inflammatory factor and oxidative stress indexes. Immunohistochemistry, western blot assay and reverse transcription-quantitative polymerase chain reaction were applied to detect calcium channel protein expression in the uterus. Western blot analysis was
\end{abstract}

Correspondence to: Dr Jun Wang, Department of Maternity, General Hospital of Shenyang Military Area Command, 83 Wenhua Road, Shenyang, Liaoning 110016, P.R. China

E-mail:wj202fck@163.com

Dr Mingxiao Hou, Department of Cardiothoracic Surgery, General Hospital of Shenyang Military Area Command, 83 Wenhua Road, Shenyang, Liaoning 110016, P.R. China

E-mail: houmingxiao188@163.com

"Contributed equally

Key words: S-adenosyl methionine, transient receptor protein 3, infectious premature delivery, calcium channel, transient receptor protein 3/protein kinase $\mathrm{C} \beta / \mathrm{C}$-kinase-activated protein phosphatase-1 inhibitor of $17 \mathrm{kDa}$ signaling pathway employed to measure the expression of TRPC3/PKC $\beta / \mathrm{CPI}-17$ signaling pathway-related proteins. TRPC 3 was highly expressed in the uterus of rat models of premature delivery induced by LPS. Following treatment with SAMe, inflammatory cell infiltration markedly reduced in the uterus and the tension of in vitro uterine muscle strips significantly decreased. SAMe treatment suppressed inflammatory reaction and oxidative stress, and diminished L-type and T-type calcium channel protein expression. TRPC3/PKC $\beta / C P I-17$ signaling pathway-related protein expression was also reduced. When TRPC3 expression was suppressed, the effects of SAMe against inflammation and oxidative stress were diminished. TRPC3/PKC $\beta / C P I-17$ signaling pathway-related protein expression significantly increased. SAMe was able to reduce inflammatory reaction and oxidative stress in the uterus of rat model of infectious premature delivery induced by LPS, prolong delivery time, reduce the mortality rate of offspring rats, and serve a therapeutic role. This effect is likely achieved via the regulation of uterine contractions and childbirth through the TRPC3/PKC $\beta / C P I-17$ signaling pathway.

\section{Introduction}

Premature delivery is a common complication of pregnancy. The World Health Organization reported in 2013 that $\sim 15$ million premature babies are born every year worldwide (1). Each year, $>1$ million premature babies succumb to premature complications, and many surviving children also suffer from defects in the nervous system, respiratory tract, and digestive tract (2). It has previously been demonstrated that infection is the most prevalent cause of premature delivery, accounting for $\sim 40 \%$ (3). The placenta is the intermediary between the mother and the fetus, and has an important barrier effect on the mother-fetus interface. Once bacterial endotoxin lipopolysaccharide (LPS) enters the organism, it can cause placental infection, and a number of pathological changes occur in the placenta (4). Under normal conditions, extracellular $\mathrm{Ca}^{2+}$ primarily flows through calcium channels in the cell membrane, triggering the release of $\mathrm{Ca}^{2+}$ via 
calcium release channels in the sarcoplasmic reticulum (5-7). Thus, increased intracellular $\mathrm{Ca}^{2+}$ concentrations may result in uterine contraction and premature delivery. Taken together, calcium channels serve an important role in regulating smooth muscle activity. Pyr3 is a transient receptor protein 3 (TRPC3) channel blocker, which potentiates dexamethasone sensitivity and apoptosis in acute lymphoblastic leukemia cells by disturbing $\mathrm{Ca}^{+}$signaling, mitochondrial membrane potential changes and reactive oxygen species production (8). TRPC is a non-selective cation channel composed of four monomers with six transmembrane helices, participates in many pathophysiological processes, and can transport sodium, calcium and magnesium ions $(9,10)$. When cellular calcium stores are depleted, the TRPC channel on the cell membrane is opened, resulting in the influx of extracellular $\mathrm{Ca}^{2+}$ and calcium stores are restored. This $\mathrm{Ca}^{2+}$ influx is known as store-operated calcium entry (11). Protein kinase $C \beta$ (РKC $\beta) / C$-kinase-activated protein phosphatase-1 inhibitor of $17 \mathrm{kDa}(\mathrm{CPI}-17)$ is a $\mathrm{Ca}^{2+}$-activated phospholipid-dependent protein kinase that regulates different cell functions, such as gene expression, cell proliferation, apoptosis and cell migration $(12,13)$. Myosin is a basic constituent of a thick filament and is a hexamer of macromolecular proteins consisting of two myosin heavy chains and four myosin light chains (MLC) (14). Ozaki et al (15) previously demonstrated that a PKC agonist could significantly increase the phosphorylation of MLC and the contraction of vascular smooth muscle. However, PKC agonists cannot increase the phosphorylation level of MLC20 and the contractile function of vascular smooth muscle (16). PKC $\beta / C P I-17$ serves a critical role in the calcium sensitization mechanism of smooth muscle (15).

As an organic nutrient, if amino acids can be used as therapeutic agents, it is relatively safe and reliable, has no obvious side effects, and has no adverse effects on fetal development, intelligence and heredity (17). Therefore, the use of amino acids is of great clinical value. It has previously been demonstrated that S-adenosyl methionine (SAMe) is an important metabolic intermediate in methionine, a methyl donor for the catalytic reaction of $>100$ different methyltransferases in vivo, and serves an important role in the metabolism of living cells (18). At present, SAMe is used in clinic settings for intrahepatic cholestasis and fatty liver in pregnancy, effectively improves the clinical symptoms and outcomes of pregnant women, and reduces the rate of premature delivery (19). Furthermore, SAMe is also a prescription drug for arthritis, has few side effects and has exhibited greater efficacy than conventional clinical drugs, such as indomethacin $(20,21)$. Recent studies also demonstrated that SAMe has antidepressant and antitumor effects $(22,23)$.

The present study sought to establish rat models of premature delivery induced by LPS, to investigate the effects of SAMe on infectious premature inflammatory factors and uterine contraction, and to further explore the underlying mechanism of the TRPC $3 / \mathrm{PKC} \beta / \mathrm{CPI}-17$ signaling pathway following intervention with a TRPC3 inhibitor.

\section{Materials and methods}

Animals and ethical approval. A total of 45 female and 30 male specific-pathogen-free Sprague-Dawley rats (weight,
220-260 g; age, 7 weeks) were provided by the Experimental Animal Center of the General Hospital of Shenyang Military Area Command [Shenyang, China; license no. SCXK (JUN) 2012-0002]. Female and male rats were placed in the same cage overnight at a ratio of 2:1 (3 rats per cage). Animals were housed at a constant temperature $\left(22 \pm 1^{\circ} \mathrm{C}\right)$ with $50 \%$ humidity in a $12 \mathrm{~h} \mathrm{light/dark} \mathrm{cycle.} \mathrm{The} \mathrm{rats} \mathrm{had} \mathrm{access} \mathrm{to} \mathrm{food} \mathrm{and} \mathrm{auto-}$ claved water ad libitum. The next morning, the detection of sperm on the vaginal plug or vaginal smear was considered as the day 0 of pregnancy. All animal procedures were approved by the Animal Experiments Ethics Committee of the General Hospital of Shenyang Military Area Command. Pregnant rats were randomly assigned to the control group ( $n=9)$, LPS group (model of infectious premature delivery; $n=12$ ), SAMe group (SAMe + LPS; n=12), and Pyr3 group (Pyr3 + SAMe + LPS; $\mathrm{n}=12$ ).

Preparation of rat models of infectious premature delivery and sample collection. LPS salt solution $(100 \mu \mathrm{g} / \mathrm{kg}$; cat. no. L8880; Beijing Solarbio Science \& Technology Co., Ltd., Beijing, China; saline solution was used as a solvent) was intraperitoneally administered to pregnant rats in the LPS group at day 15 of pregnancy. In the SAMe group, $30 \mathrm{mg} / \mathrm{kg}$ SAMe (physiological saline was used as the solvent; cat. no. S9990; Beijing Solarbio Science \& Technology Co., Ltd.) was intraperitoneally injected at $0.5 \mathrm{~h}$ following LPS injection. In the Pyr3 group, 30 mg/kg Pyr (Sigma-Aldrich; Merck KGaA, Darmstadt, Germany) was intraperitoneally administered at 30 min following SAMe injection. In the control group, an equal volume of physiological saline was intraperitoneally administered concurrently with LPS administration in the other groups. The occurrence of premature delivery $(<19$ days of pregnancy) was recorded. Following premature delivery, rats were anesthetized with $2 \%$ sodium pentobarbital $(40 \mathrm{mg} / \mathrm{kg}$; Beijing Huaye Huanyu Chemical Co., Ltd., Beijing, China). Arterial blood was collected and $\sim 5 \mathrm{ml}$ serum was isolated by centrifugation at $1,000 \mathrm{x}$ g for $5 \mathrm{~min}$ at $4^{\circ} \mathrm{C}$. The blood plasma was isolated, packed separately and stored at $-80^{\circ} \mathrm{C}$ for ELISA. Rats were subsequently sacrificed via anesthesia overdose with $2 \%$ sodium pentobarbital $(120 \mathrm{mg} / \mathrm{kg})$. The route of sodium pentobarbital administration for anesthesia and sacrifice was intraperitoneal injection. The uterus and placenta was harvested and adipose tissue was removed. Blood was washed away with physiological saline. A random section of each sample was fixed in $10 \%$ paraformaldehyde at room temperature for $48 \mathrm{~h}$ and the other was stored in liquid nitrogen.

Determination of latency and live birth rate in each group. Following the evaluation of premature delivery in each group, latency (the time between LPS administration and the first fetus delivery) and live birth rate in each group were recorded.

Determination of muscle tension of uterine muscle strips of pregnant rats. Uterus tissue samples $(\sim 1 \mathrm{~cm}$ longitudinal direction) were washed with physiological saline, fixed on the specimen hook of a multi-channel physiological signal acquisition system, and placed in a tank filled with $8 \mathrm{ml}$ Tyrode's solution. The sample was also connected to a tension converter to record uterine contraction. 
Hematoxylin and eosin (HE) staining. Rat uterus samples were dehydrated with a graded alcohol series, permeabilized with xylene, immersed and embedded in paraffin, and cut into $5-\mu$ m-thick sections. Sections were stained with hematoxylin for $5 \mathrm{~min}$ at room temperature, washed with PBS, differentiated with hydrochloric acid ethanol for $3 \mathrm{sec}$, stained with eosin for $1 \mathrm{~min}$ at room temperature and mounted with neutral resin. Changes in rat uterine tissue were observed under light microscopy (magnification, x12.6).

Immunohistochemistry. Paraffin-embedded 5- $\mu$ m-thick sections were placed in a $67^{\circ} \mathrm{C}$ oven for $2 \mathrm{~h}$, dewaxed, hydrated in a descending alcohol series and treated with citrate buffer (pH, 6.0; cat. no. C1010; Beijing Solarbio Science \& Technology Co., Ltd.) at $100^{\circ} \mathrm{C}$ for $10 \mathrm{~min}$. Antigen retrieval was performed via microwave heating at $100^{\circ} \mathrm{C}$. Each section was treated with $\sim 30 \mu 13 \%$ hydrogen peroxide aqueous solution (cat. no. 10011208; Sinopharm Chemical Reagent Co., Ltd., Shanghai, China) at room temperature for $10 \mathrm{~min}$, and blocked with $5 \%$ bovine serum albumin for $45 \mathrm{~min}$ at room temperature. After abundant liquid was discarded, sections were incubated with antibodies against TRPC3 (ab51560; rabbit; $97 \mathrm{kDa}$; 1:500; Abcam, Cambridge, UK); calcium channel, voltage-dependent, L type, $\alpha$ 1C subunit (Cav1.2; ab58552; rabbit; 249 kDa; 1:1,000; Abcam); calcium channel, voltage-dependent, T type, $\alpha 1 \mathrm{G}$ subunit (Cav3.1; ab95092; rabbit; 262 kDa; 1:1,000; Abcam); and calcium channel, voltage-dependent, $\mathrm{T}$ type, $\alpha 1 \mathrm{H}$ subunit (Cav3.2; ab95092; rabbit; $262 \mathrm{kDa} ; 1: 1,000 \mathrm{Abcam})$ at $4^{\circ} \mathrm{C}$ overnight. Sections were subsequently incubated with horseradish peroxidase (HRP)-conjugated anti-goat immunoglobulin G secondary antibodies (1:1,000; cat. no. ab6721; Abcam) for $2 \mathrm{~h}$ at room temperature, washed with PBS, visualized with 3,3'-diaminobenzidine, counterstained with hematoxylin for $2 \mathrm{~min}$ at room temperature, dehydrated through a graded alcohol series, permeabilized with xylene, mounted with neutral resin and observed using a light microscope (magnification, x25.2).

ELISA. The following ELISA kits were used to analyze rat serum samples, according to the manufacturers' protocols: Interleukin (IL)-1 $\beta$ (SEA563Ra; Uscn Life Sciences, Inc., Wuhan, China), IL-8 (H008; Nanjing Jiancheng Bioengineering Institute, Nanjing, China), tumor necrosis factor $\alpha$ (TNF- $\alpha$; SEA133Ra; Uscn Life Sciences, Inc.), IL-10 (SEA056Ra; Uscn Life Sciences, Inc.), malondialdehyde (MDA; A003-2; Nanjing Jiancheng Bioengineering Institute), superoxide dismutase (SOD; A001-3; Nanjing Jiancheng Bioengineering Institute) and nitric oxide (NO; A013-2; Nanjing Jiancheng Bioengineering Institute). Blank wells, standard wells and detected wells were used. Blank wells contained $100 \mu \mathrm{l}$ PBS; the other wells contained $100 \mu \mathrm{l}$ standard preparation (provided with the kits) or rat serum samples. All were incubated at $37^{\circ} \mathrm{C}$ for $2 \mathrm{~h}$. Following removal of the liquid, the plate was washed three times with the reagent provided by the kits. HRP-conjugated fluid $(100 \mu \mathrm{l})$ was added to each well at $37^{\circ} \mathrm{C}$ for $1 \mathrm{~h}$. Following removal of the liquid, sections were dried, and $100 \mu \mathrm{l}$ substrate solution was added to each well and incubated at $37^{\circ} \mathrm{C}$ in the dark for $\sim 15 \mathrm{~min}$. Stop buffer $(50 \mu \mathrm{l})$ was subsequently added to each well. Optical density values were measured in each well at $450 \mathrm{~nm}$ using a microplate reader.
Western blot analysis. Rat placenta was lysed in radioimmunoprecipitation assay lysate containing protease inhibitor (cat. no. R0010; Beijing Solarbio Science \& Technology Co., Ltd.) on ice for $30 \mathrm{~min}$. Following centrifugation at 4,000 x $\mathrm{g}$ for $20 \mathrm{~min}$ at $4^{\circ} \mathrm{C}$, supernatant was collected. Proteins were quantified using a Bicinchoninc Acid Assay Protein Quantification kit. The protein samples (30 $\mu \mathrm{g} / \mathrm{lane}$ ) were subjected to $10 \%$ SDS-PAGE, and transferred onto polyvinylidene fluoride membranes. The membrane was blocked with 5\% skimmed milk for $2 \mathrm{~h}$ at room temperature, and incubated with antibodies against Cav1.2 (1:1,000), Cav3.1 (1:1,000), Cav3.2 (1:1,000), PKC $\beta$ (ab227490; rabbit; 76 kDa; 1:1,000; Abcam) CPI-17 (ab32213; rabbit; 17 kDa; 1:1,000; Abcam), phosphorylated (p-)CPI-17 (ab52174; rabbit; 17 kDa; 1:1,000; Abcam) and GAPDH (ab181602; rabbit; 36kDa; 1:10,000; Abcam) at $4^{\circ} \mathrm{C}$ overnight. Subsequently, the membrane was washed with TBST, and incubated with HRP-conjugated secondary antibody (1:2,000; cat. no. ab6721; Abcam) at room temperature for $2 \mathrm{~h}$. Proteins were visualized using an enhanced chemiluminescence kit and gel imaging system. Absorbance values were analyzed using Image Tools (Image J 1.8.0; National Institutes of Health, Bethesda, MD, USA).

Reverse transcription-quantitative polymerase chain reaction (RT-qPCR). Rat uterus samples were triturated and TRIzol reagent (15596018; Life Technologies; Thermo Fisher Scientific, Inc., Waltham, MA, USA) was used to extract RNA, according to the manufacturer's protocol. RNA was reverse-transcribed into cDNA using a High-Capacity RNA-to-cDNA kit (4387406; Invitrogen; thermo Fisher Scientific, Inc.). qPCR was performed using a QuantiFast SYBR Green PCR kit (204057; Qiagen GmbH, Hilden, Germany) according to the manufacturer's protocol. Relative gene expression data was quantified using the $2^{-\Delta \Delta \mathrm{Cq}}$ method (24). GAPDH was used as the reference gene. Primers used for $\mathrm{qPCR}$ are presented in Table I.

Statistical analysis. All data were statistically analyzed using SPSS 19.0 (IBM Corp., Armonk, NY, USA). Data are presented as the mean + standard deviation. One-way analysis of variance followed by Tukey's post-hoc test was used for comparison among groups. All experiments were repeated in triplicate. $\mathrm{P}<0.05$ was considered to indicate a statistically significant difference.

\section{Results}

Therapeutic effect of SAMe on infectious premature delivery. The effect of SAMe on rats with infectious premature delivery was assessed as latency and live birth rate (Fig. 1A and B). In the control group, latency time was $\sim 70.4 \mathrm{~h}$, or $\sim 19$ days of pregnancy, with a live birth rate of $96 \%$. In the LPS group, the fetus was delivered within $24 \mathrm{~h}, \sim 15 \mathrm{~h}$, with a live birth rate of $4.3 \%$. These results indicated the model of infectious premature delivery was established successfully. In the SAMe group the latency time was $59.7 \mathrm{~h}$ and the live birth rate was $72.5 \%$. However, the therapeutic effect of SAMe was significantly reduced following addition of Pyr3. Compared with the LPS group, latency was prolonged by $\sim 28.9 \mathrm{~h}$, and the live birth rate was $43.5 \%$. 
Table I. Gene primers used in reverse transcription-quantitative polymerase chain reaction.

\begin{tabular}{|c|c|}
\hline Gene & Primer sequence $\left(5^{\prime}-3^{\prime}\right)$ \\
\hline \multirow[t]{2}{*}{ Cav1.2 } & Forward: CGGATCTGGAAGCTCGGAT \\
\hline & Reverse: GTGATTGCGGAGCCCGA \\
\hline \multirow[t]{2}{*}{ Cav3.1 } & Forward: TTAAGAGCTACCTGATCGAG \\
\hline & Reverse: TGTATCCGCACCTTCTGCA \\
\hline \multirow[t]{2}{*}{ Cav3.2 } & Forward: GCCATTCTCTCCTTCCTGCA \\
\hline & Reverse: CGCAGCAGCAAATTTATG \\
\hline \multirow[t]{2}{*}{ GAPDH } & Forward: GCATGATGCCGGCAGCTTT \\
\hline & Reverse: CAGCAACTGAATGAGGCCA \\
\hline
\end{tabular}

Cav1.2, calcium channel, voltage-dependent, L type, $\alpha 1 \mathrm{C}$ subunit; Cav3.1, calcium channel, voltage-dependent, T type, $\alpha$ 1G subunit; Cav3.2, calcium channel, voltage-dependent, T type, $\alpha 1 \mathrm{H}$ subunit.

SAMe suppresses inflammatory factors $I L-1 \beta, I L-8, T N F-a$, $I L-10, M D A, S O D$ and NO serum content. To verify the effect of SAMe against inflammatory reaction and oxidative stress, rat serum was collected for ELISA (Fig. 2A-D). Results demonstrated that IL-1 $\beta$, IL- 8 and TNF- $\alpha$ expression was significantly increased in the LPS group $(\mathrm{P}<0.05$ vs. control), whereas IL-10 expression was significantly reduced $(\mathrm{P}<0.05$ vs. control). Following treatment with SAMe, IL-1 $\beta$, IL-8 and TNF- $\alpha$ expression was significantly diminished $(\mathrm{P}<0.05$ vs. LPS), whereas IL-10 expression significantly increased $(\mathrm{P}<0.05$ vs. LPS). Following treatment with Pyr3, the anti-inflammatory effect of SAMe was significantly reduced; IL-1 $\beta$, IL- 8 and TNF- $\alpha$ expression significantly increased $(\mathrm{P}<0.05$ vs. SAMe) and IL-10 expression significantly decreased $(\mathrm{P}<0.05$ vs. $\mathrm{SAMe}$ ). These data suggest that SAMe treatment significantly reduces the inflammatory reaction induced by LPS.

Further detection of serum oxidative stress-related factors (Fig. 2E-G) revealed that MDA and NO expression was significantly increased in the LPS group $(\mathrm{P}<0.05$ vs. control), and SOD expression was significantly diminished $(\mathrm{P}<0.05$ vs. control). Following SAMe treatment, MDA and NO expression was significantly reduced $(\mathrm{P}<0.05$ vs. LPS $)$, and SOD expression was significantly increased $(\mathrm{P}<0.05$ vs. LPS). Following treatment with Pyr3, MDA and NO expression was significantly increased $(\mathrm{P}<0.05$ vs. SAMe), while SOD expression was significantly reduced ( $\mathrm{P}<0.05 \mathrm{vs}$. SAMe). These data indicate that SAMe can significantly inhibit LPS-induced inflammatory reaction and oxidative stress. Following inhibiting TRPC3, the effect of SAMe was significantly weakened. These findings suggest that SAMe exerted its effect through the TRPC3 signaling pathway.

SAMe improves muscle tension of the uterus. H\&E staining was utilized to observe the pathological changes in rat uterus (Fig. 3A). Results indicated that following LPS-induced premature delivery, in the LPS group, a large number of inflammatory cells infiltrated into the smooth muscle layer of uterus in rats, and cells were irregularly distributed. Following SAMe treatment, this inflammatory cell infiltration was markedly reduced. Following suppressing TRPC3 expression with Pyr3, inflammatory cell infiltration markedly increased in the smooth muscle layer of rat uterus.

Muscle tension of the rat uterus (Fig. 3B) indicated that following LPS-induced premature delivery, muscle tension of in vitro uterine muscle strips significantly increased $(\mathrm{P}<0.05$ vs. control). The muscle tension in the SAMe group significantly diminished $(\mathrm{P}<0.05$ vs. LPS). Following suppressing TRPC 3 , muscle tension significantly increased $(\mathrm{P}<0.05$ vs. SAMe).

SAMe reduces the expression of calcium channel in rats with infectious premature delivery. Western blot analysis (Fig. 4A) was used to measure Cav1.2, Cav3.1 and Cav3.2 expression in the rat uterus. Data suggested that Cav1.2, Cav3.1 and Cav3.2 expression increased in rats with premature delivery induced by LPS (all, $\mathrm{P}<0.05$ vs. control). Following treatment with SAMe, Cav1.2, Cav3.1 and Cav3.2 expression significantly decreased (all, P<0.05 vs. LPS). Following inhibition of TRPC3, Cav1.2, Cav3.1 and Cav3.2 expression significantly increased (all, $\mathrm{P}<0.05$ vs. SAMe). RT-qPCR (Fig. 4B) results were consistent with western blot assay results. Immunohistochemical analysis (Fig. 4C) indicated that compared with the control group, Cav1.2, Cav3.1 and Cav3.2 expression notably increased in rats with premature delivery induced by LPS. Following treatment with SAMe, Cav1.2, Cav3.1 and Cav3.2 expression significantly decreased compared with the LPS group. Following inhibition of TRPC3, Cav1.2, Cav3.1 and Cav3.2 expression significantly increased compared with the SAMe group. Immunostaining was indicated with a blue color. These findings suggest that L-type calcium channels and T-type calcium channels participate in the occurrence of premature delivery. SAMe can effectively weaken the expression of L-type and T-type calcium channel-related proteins and uterine contraction.

SAMe promotes uterine contraction and delivery via the TRPC3/PKC $/$ /CPI-17 signaling pathway. Western blotanalysis was utilized to measure the expression of TRPC $3 / \mathrm{PKC} \beta / \mathrm{CPI}-17$ signaling pathway-related proteins in the rat uterus (Fig. 5). Data suggested that following premature delivery induced by LPS, PKC $\beta$ and p-CPI-17 expression significantly increased $(\mathrm{P}<0.05$ vs. control). Following treatment with $\mathrm{SAMe}, \mathrm{PKC} \beta$ and $\mathrm{p}-\mathrm{CPI}-17$ expression significantly diminished $(\mathrm{P}<0.05$ vs. LPS). These data indicate that the TRPC $3 / \mathrm{PKC} \beta / \mathrm{CPI}-17$ signaling pathway participated in the occurrence and development of premature delivery. Following the application of Pyr3, inflammatory factor expression increased, and PKC $\beta$ and p-CPI-17 expression significantly increased $(\mathrm{P}<0.05$ vs. $\mathrm{SAMe})$. These results indicate that the anti-inflammatory effect of SAMe is exerted via the TRPC3/PKC $/$ CPI-17 signaling pathway.

\section{Discussion}

Infection has been demonstrated to be associated with premature delivery. Histological evidence of intrauterine infection is exhibited in $19-74 \%$ preterm infants (25). At present, it is believed that infectious preterm delivery is associated with Gram-negative bacteria endotoxin LPS that stimulates an increase in local cytokines, and subsequently stimulates 
A

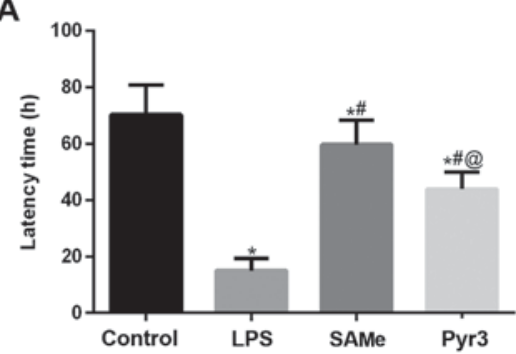

B

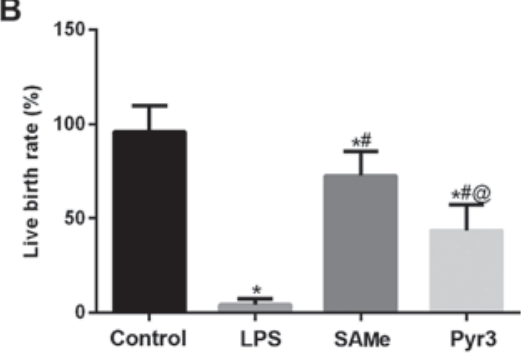

Figure 1. Therapeutic effect of SAMe on infectious premature delivery. Following establishing the infectious premature delivery models in rats, (A) latency and (B) live birth rate were observed. ${ }^{*} \mathrm{P}<0.05$ vs. control group; ${ }^{\#} \mathrm{P}<0.05$ vs. LPS group; ${ }^{\circledR} \mathrm{P}<0.05$ vs. SAMe group. SAMe, S-adenosyl methionine; LPS, lipopolysaccharide. Data are provided as the mean \pm standard deviation $(n=45)$.

abnormal uterine contraction (26). In the present study, pregnant rat models of premature delivery were successfully established via LPS administration. SAMe treatment revealed that SAMe could reduce the rate of premature delivery, elevate survival rate of fetal rats, improve muscle tension, and increase calcium-specific protein expression, indicating that $\mathrm{SAMe}$ has a therapeutic effect on infectious premature delivery. Administration of the TRPC3 protein inhibitor Pyr3 demonstrated that the therapeutic effect of SAMe was suppressed, and that the TRPC3/PKC $\beta / C P I-17$ signaling pathway has an important regulatory effect.

SAMe is a physiologically active substance found in all tissues and fluids, and is converted from methionine under the action of adenosylmethionase (18). As a methyl donor and a physiological sulfur compound, SAMe can participate in important biochemical reactions in the human body and transform thioxo into taurine (18). Previous studies have also verified that SAMe could regulate IL-10 production in monocytes, and increase IL-6 synthesis in monocytes and liver Kupffer cells following LPS stimulation $(27,28)$. Its metabolite, 5-methylthioadenosine, has an important immunomodulatory effect on the inflammatory response of hepatocytes (29). Simultaneously, SAMe is able to reduce serum TNF- $\alpha$ and TGF- $\beta$ contents $(30,31)$. In the present study, SAMe treatment increased IL-1 $\beta$, IL- 8 , TNF- $\alpha$ and IL-10 contents in rats with infectious premature delivery, and had an inhibitory effect on oxidative stress factors. These findings indicated that SAMe could suppress inflammatory reaction and the resulting stress response in rats with infectious premature delivery.

The mechanism of infectious premature delivery is complex and is the result of a number of factors. There are many theories about the causes of premature childbirth, including neurotransmitter theory, mechanical theory, hormone control theory and immunity theory (32-34). These theories share a common final path; the induction of the contraction of uterine smooth muscle (35). The contraction of the uterus is determined by the concentration of free calcium in the muscle cells (36). Prior to uterine contraction, cytoplasmatic free calcium concentration increases significantly. Mark et al (37) recently presented a mechanism of intracellular calcium signaling that leads to cyclic uterine contractions. Normally, extracellular calcium ions flow through the calcium channel of the cell membrane, triggering $\mathrm{Ca}^{2+}$ release from the sarcoplasmic reticulum calcium release channel. Therefore, elevated intracellular calcium concentration results in uterine contraction and premature delivery. It can be observed that the calcium channel serves an important role in regulating smooth muscle activity. The present study detected the expression of L-type calcium channel Cav1.2 and T-type calcium channel Cav3.1 and Cav3.2 expression in the rat uterus $(38,39)$. Results indicated that each protein expression increased in premature delivery in rats of the LPS group, and confirmed that L-type and T-type calcium channels participated in the occurrence of premature delivery. The addition of SAMe revealed that calcium channel protein expression decreased in each group, and muscle tension test verified this conclusion. It is thus clear that SAMe could effectively weaken L-type and T-type calcium channel-related protein expression, and weaken uterine contraction.

TRPC channel has an extensive physiological role in addition to mediating extracellular calcium influx and inducing uterine contraction, which has been confirmed in previous studies of the nervous system, vascular smooth muscle, myocardium, and skin (40-43). A variety of physiological phenomena depend on the opening of TRPC channels. A previous study verified that TRPC channel was expressed in the pregnant uterus (44). The upregulation of TRPC3 channel, and L-type and T-type calcium channel functions enhances the contractility of uterine spiral arteries during pregnancy, which is associated with labor onset. In the present study, on the basis of SAMe treatment, Pyr3 was administered, so that the inhibitory effect of SAMe on inflammation and regulatory effect on calcium channels were suppressed, indicating that the therapeutic effect of SAMe on infectious premature delivery is associated with TRPC channel. It has previously been demonstrated that TRPC channels are expressed in mammalian uterine muscle tissue (45). Mechanical traction of human uterine smooth muscle ex vivo can increase the expression of TRPC 3 channel (46).

The PKC $\beta / C P I-17$ pathway serves an important role in the contraction of vascular smooth muscle and the mechanism of calcium sensitization. Su et al (47) previously induced CPI-17 gene silencing in bronchial smooth muscle using RNA interference technology, and observed that calcium sensitization, contraction frequency and contractility of bronchial smooth muscle decreased, which suggested that CPI-17 has an effect on the contraction of smooth muscle. Ozaki et al (15) previously demonstrated that PKC agonist can significantly increase the phosphorylation of MLC and the contraction of vascular smooth muscle. In the presence of the specific MLCP activity inhibitor, Rho-associated kinase, exogenous phosphorylated 
A

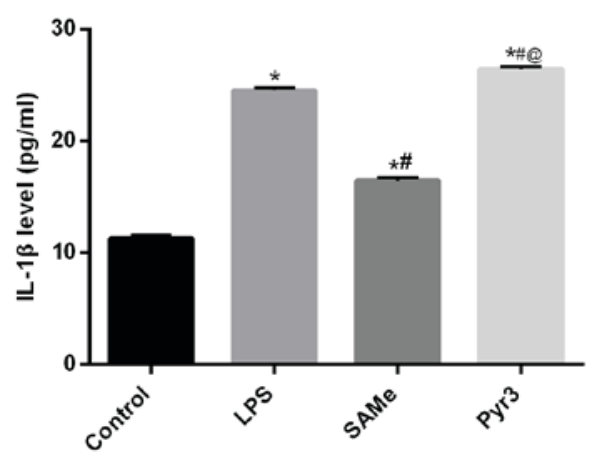

C

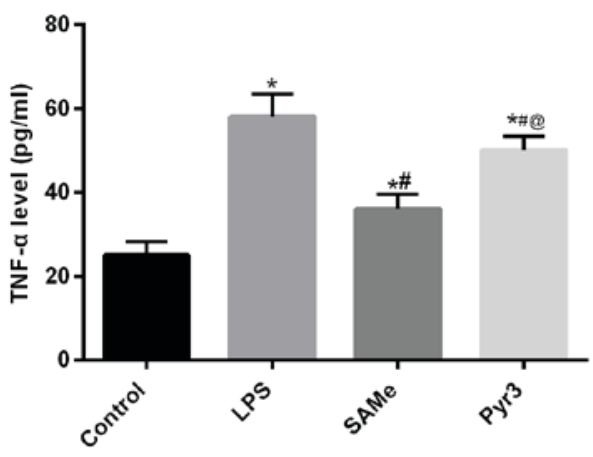

$\mathbf{E}$

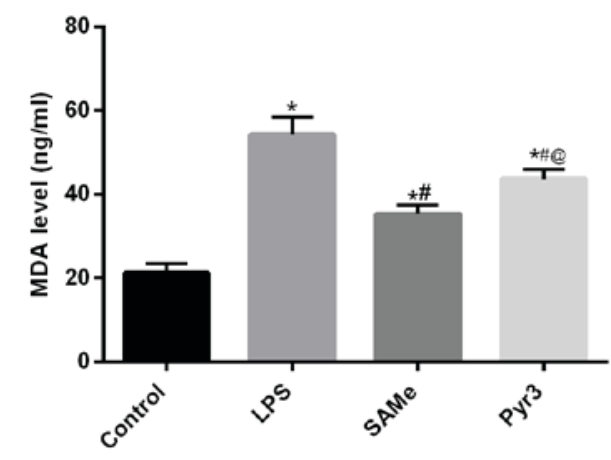

G

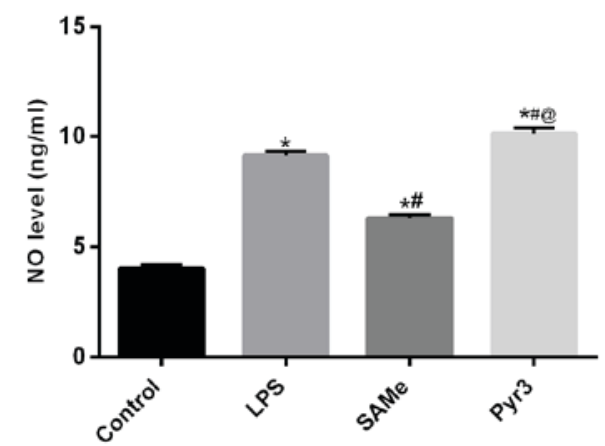

B

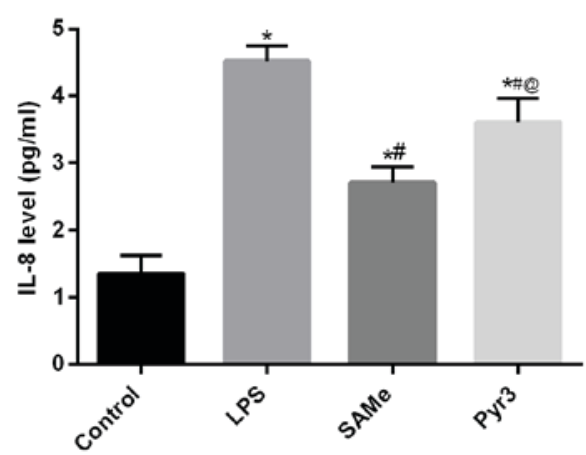

D

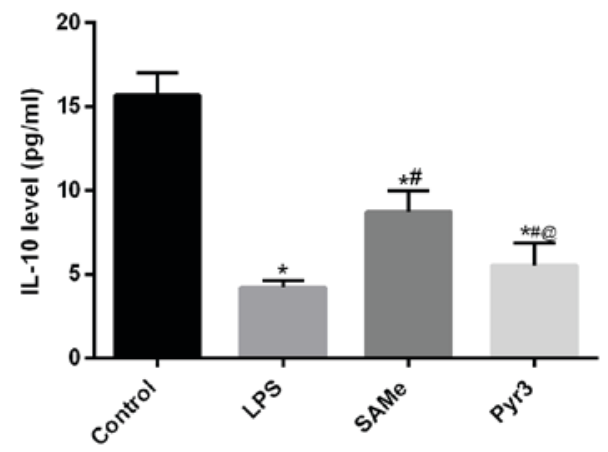

$\mathbf{F}$

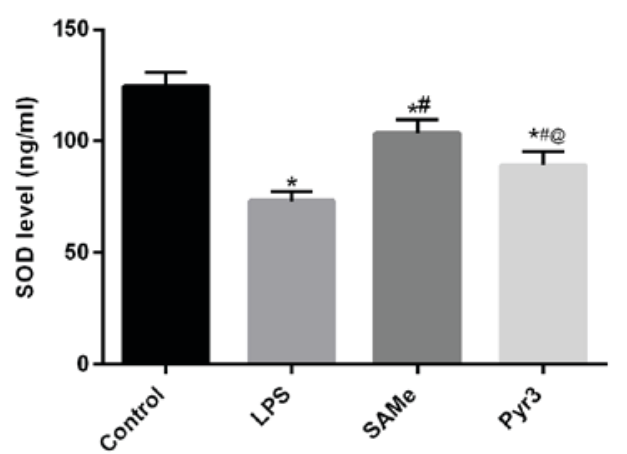

Figure 2. SAMe suppresses inflammatory factors IL-1 $\beta$, IL-8, TNF- $\alpha$, IL-10, MDA, SOD and NO. (A) IL-1 $\beta$, (B) IL-8, (C) TNF- $\alpha$, (D) IL-10, (E) MDA, (F) SOD and (G) NO serum levels were detected by ELISA. " $\mathrm{P}<0.05$ vs. control group; ${ }^{\text {P }}<0.05$ vs. LPS group; ${ }^{\circledR} \mathrm{P}<0.05$ vs. SAMe group. SAMe, S-adenosyl methionine; IL, interleukin; TNF- $\alpha$, tumor necrosis factor $\alpha$; MDA, malondialdehyde; SOD, superoxide dismutase; NO, nitric oxide; LPS, lipopolysaccharide. Data are provided as the mean \pm standard deviation $(n=45)$.

CPI-17 can increase the contraction of vascular smooth muscle in a dose-dependent manner (48). The present study detected proteins in the TRPC3/PKC $\beta / C P I-17$ signaling pathway, and demonstrated that SAMe could downregulate the increased expression of TRPC3, PKC $\beta$ and p-CPI-17 induced by infectious premature delivery. When TRPC3 inhibitor was added, 
A

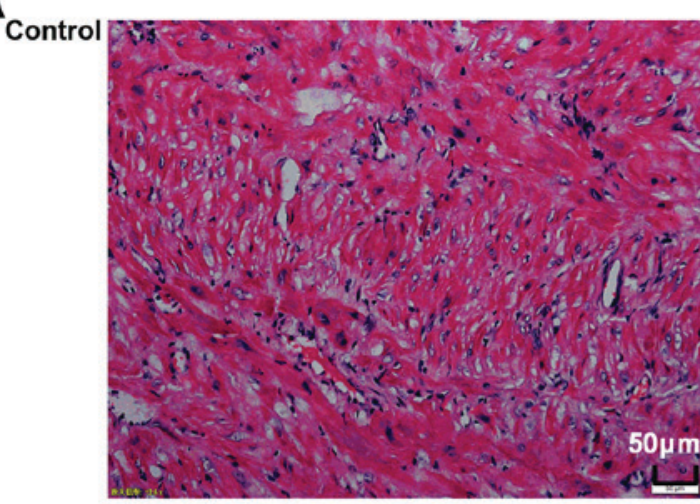

SAMe

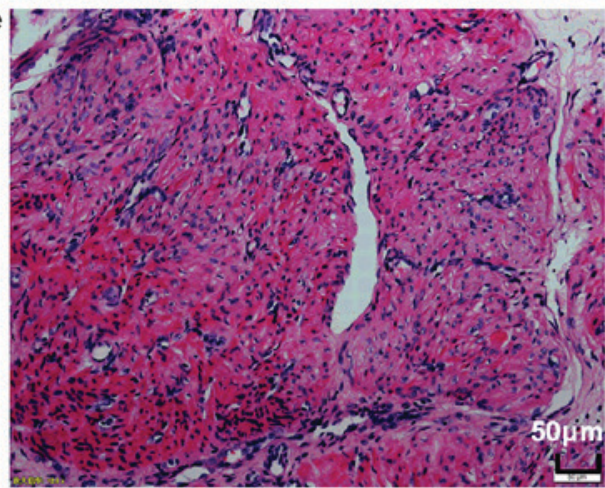

B

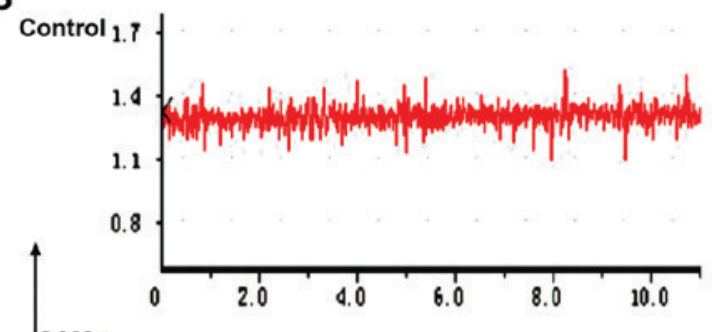

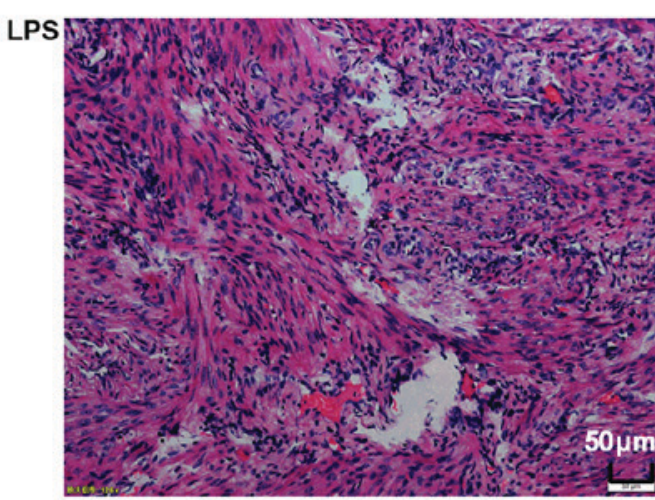

Pyr3

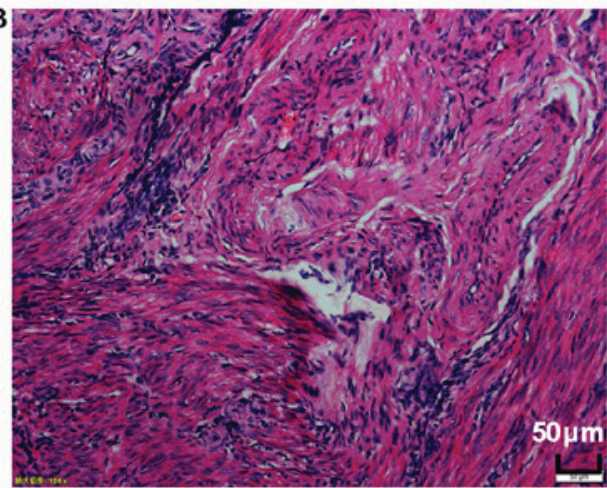

LPS

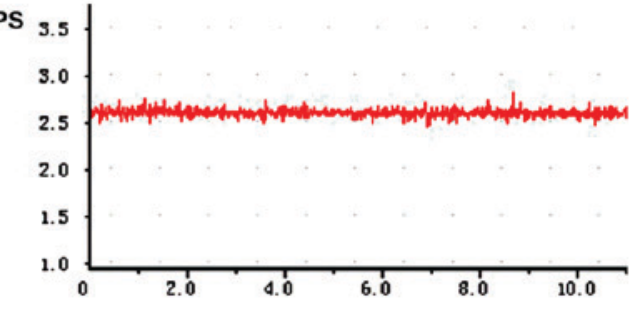

Pyr3

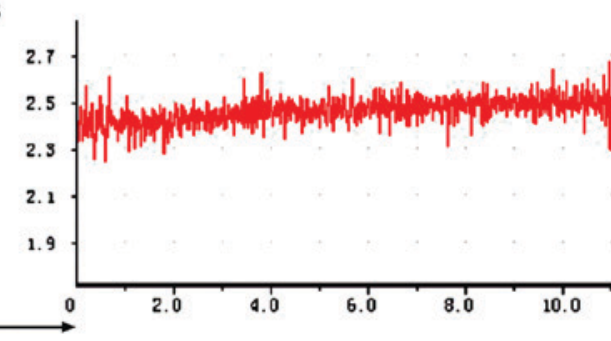

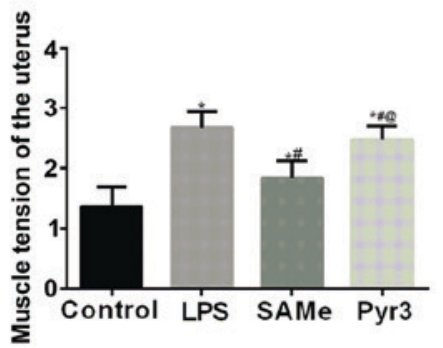

Figure 3. SAMe improves muscle tension of the uterus. (A) Hematoxylin and eosin staining. (B) Muscle tension of the rat uterus. "P<0.05 vs. control group; ${ }^{\#} \mathrm{P}<0.05$ vs. LPS group; ${ }^{\circledR} \mathrm{P}<0.05$ vs. SAMe group. SAMe, S-adenosyl methionine; LPS, lipopolysaccharide. Data are provided as the mean \pm standard deviation $(\mathrm{n}=45)$.

the effect of SAMe was no longer apparent. These data indicate that SAMe improves muscle tension, suppresses the influx of exogenous calcium ions, and inhibits inflammatory reaction through the TRPC $3 / \mathrm{PKC} \beta / \mathrm{CPI}-17$ pathway, thereby ameliorating infectious premature delivery. The present study is a preliminary exploration of the mechanism of action of SAMe, and the underlying regulatory mechanism in improving infectious premature delivery requires further investigations. 
A
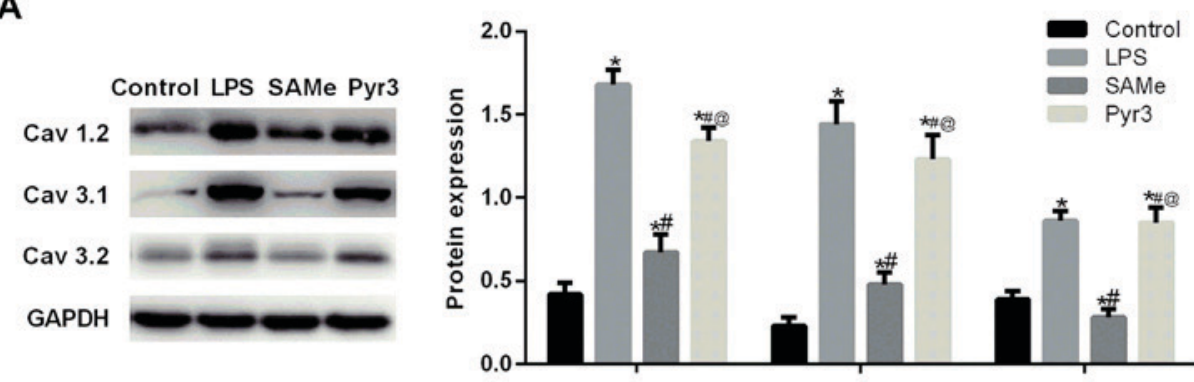

B

Cav 1.2

Cav 3.1

Cav 3.2

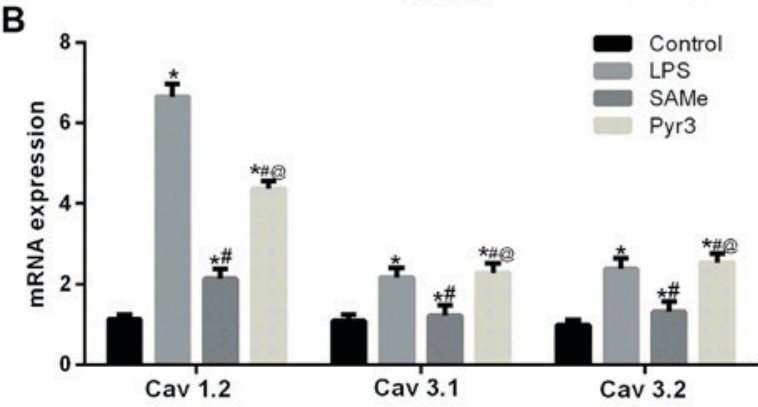

C

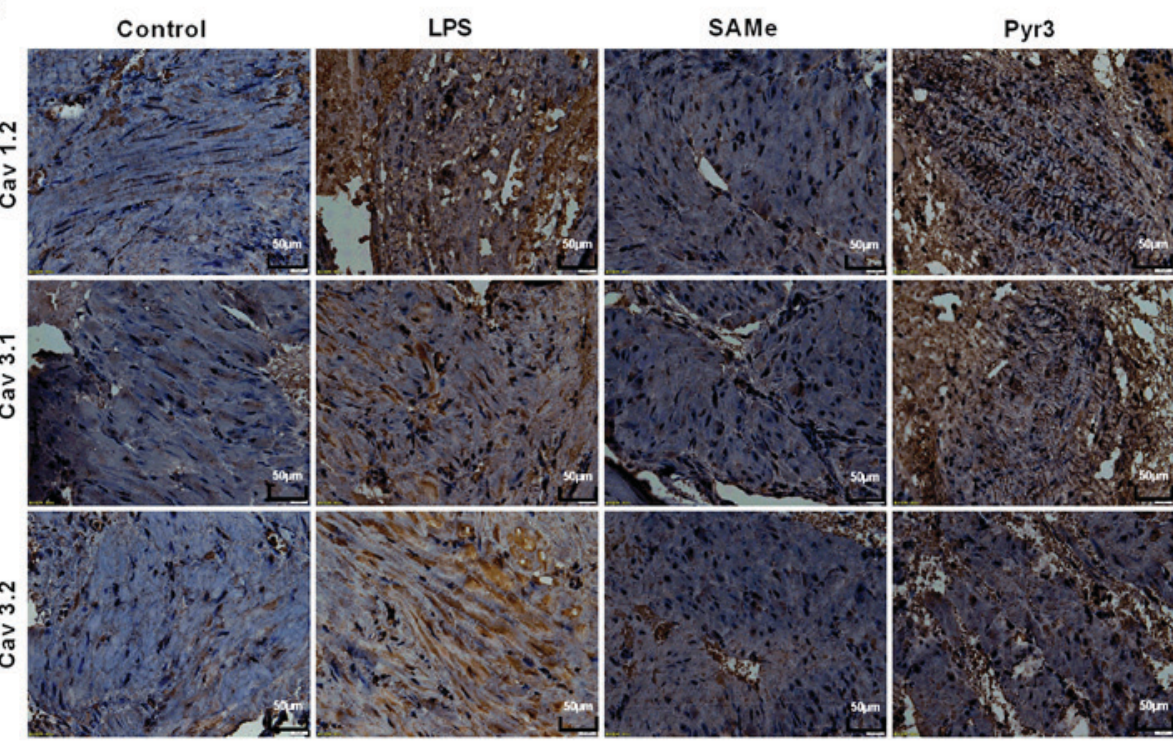

Figure 4. SAMe reduces the expression of calcium channels in rats with infectious premature delivery. Cav1.2, Cav3.1 and Cav3.2 expression were detected by (A) western blot analysis, (B) reverse transcription-quantitative polymerase chain reaction and (C) immunohistochemistry. "P<0.05 vs. control group; ${ }^{\prime \prime} \mathrm{P}<0.05$ vs. LPS group; ${ }^{\circledR} \mathrm{P}<0.05$ vs. SAMe group. SAMe, S-adenosyl methionine; Cav1.2, calcium channel, voltage-dependent, L type, $\alpha$ 1C subunit; Cav3.1, calcium channel, voltage-dependent, $\mathrm{T}$ type, $\alpha$ G subunit; Cav3.2, calcium channel, voltage-dependent, $\mathrm{T}$ type, $\alpha$ 1H subunit; LPS, lipopolysaccharide. Data are provided as the mean \pm standard deviation $(n=45)$.
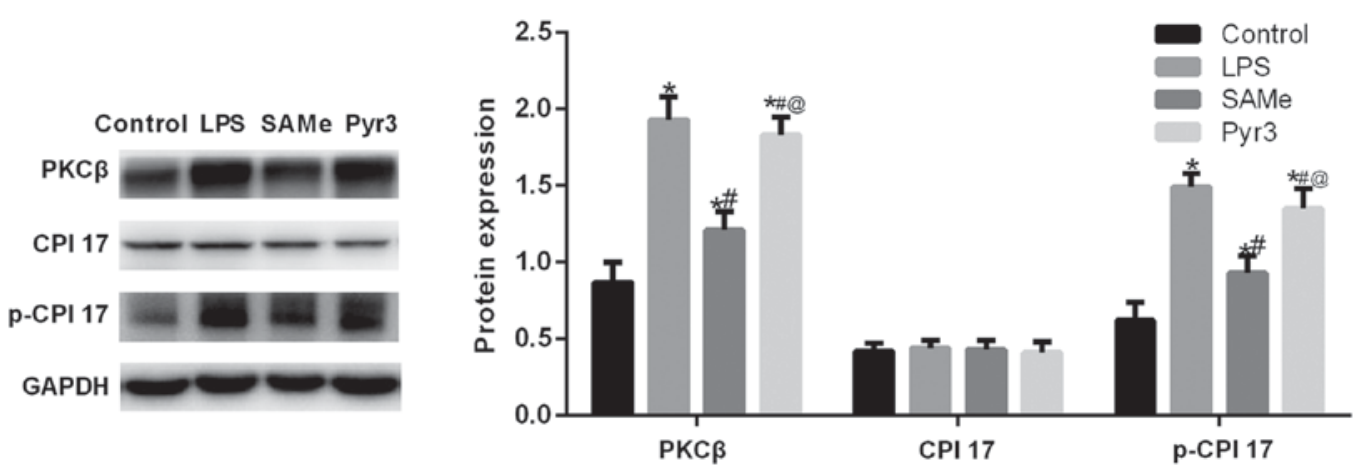

Figure 5. SAMe promotes uterine contraction and delivery through the TRPC3/PKC $\beta / C P I-17$ signaling pathway. TRPC3/PKC $\beta / C P I-17$ signaling pathway-related protein expression levels were detected by western blot analysis. ${ }^{\mathrm{P}}<0.05$ vs. control group; ${ }^{*} \mathrm{P}<0.05$ vs. LPS group; ${ }^{\circledR} \mathrm{P}<0.05$ vs. SAMe group. SAMe, S-adenosyl methionine; TRPC3, transient receptor protein 3; PKC $\beta$, protein kinase C $\beta$; CPI-17, C-kinase-activated protein phosphatase-1 inhibitor of $17 \mathrm{kDa}$; LPS, lipopolysaccharide; $\mathrm{p}$, phosphorylated. Data are provided as the mean \pm standard deviation $(\mathrm{n}=45)$. 
In conclusion, the present study successfully established a rat model of infectious premature delivery. SAMe was able to prolong the delivery time, decrease the mortality rate, inhibit the inflammatory reaction and oxidative stress, and regulate the calcium channel expression in this model. Its regulatory mechanism is possibly associated with the PKC/PLC $\beta / C P I-17$ signaling pathway. These findings provide a basis for the clinical prevention and treatment of infectious premature delivery and targeted therapy of novel drugs.

\section{Acknowledgements}

Not applicable.

\section{Funding}

The present study was supported by the Startup Foundation for Liaoning Province of China (grant no. 201601392) and the Startup Foundation for China (grant no. 2017M613440).

\section{Availability of data and materials}

The datasets used and/or analyzed during the current study are available from the corresponding author on reasonable request.

\section{Authors' contributions}

JG, TH, XL, JW and MH conceived and designed the study, acquired the data, interpreted the results and drafted the manuscript. $\mathrm{MH}$ contributed to the acquisition of funding and support. JG, TH, XL, LS and JZ performed the experiments. YH and YX analyzed the data. All authors read and approved the final manuscript.

\section{Ethics approval and consent to participate}

All animal procedures were approved by the Animal Experiments Ethics Committee of the General Hospital of Shenyang Military (Shenyang, China).

\section{Consent for publication}

Not applicable.

\section{Competing interests}

The authors declare that they have no competing interests.

\section{References}

1. Vogel JP, Chawanpaiboon S, Watananirun K, Lumbiganon P, Petzold M, Moller AB, Thinkhamrop J, Laopaiboon M, Seuc AH, Hogan D, et al: Global, regional and national levels and trends of preterm birth rates for 1990 to 2014: Protocol for development of World Health Organization estimates. Reprod Health 13: 76, 2016.

2. Ryan JG and Dogbey E: Preterm births: A global health problem. MCN Am J Matern Child Nurs 40: 278-283, 2015.

3. Rours GI, Duijts L, Moll HA, Arends LR, de Groot R, Jaddoe VW, Hofman A, Steegers EA, Mackenbach JP, Ott A, et al: Chlamydia trachomatis infection during pregnancy associated with preterm delivery: A population-based prospective cohort study. Eur J Epidemiol 26: 493-502, 2011
4. Dowling O, Chatterjee PK, Gupta M, Tam Tam HB, Xue X, Lewis D, Rochelson B and Metz CN: Magnesium sulfate reduces bacterial LPS-induced inflammation at the maternal-fetal interface. Placenta 33: 392-398, 2012.

5. Bagur R and Hajnóczky G: Intracellular $\mathrm{Ca}^{2+}$ sensing: Its role in calcium homeostasis and signaling. Mol Cell 66: 780-788, 2017.

6. Kaneko S: Cognitive Function and Calcium. Structures and functions of Ca2+-permeable channels. Clin Calcium 25: 181-187, 2015 (In Japanese).

7. Reddish FN, Miller CL, Gorkhali R and Yang JJ: Calcium dynamics mediated by the endoplasmic/sarcoplasmic reticulum and related diseases. Int J Mol Sci 18: pii: E1024, 2017.

8. Abdoul-Azize S, Buquet C, Vannier JP and Dubus I: Pyr3, a TRPC3 channel blocker, potentiates dexamethasone sensitivity and apoptosis in acute lymphoblastic leukemia cells by disturbing $\mathrm{Ca}(2+)$ signaling, mitochondrial membrane potential changes and reactive oxygen species production. Eur J Pharmacol 784: 90-98, 2016.

9. Eder P, Poteser M, Romanin C and Groschner $\mathrm{K}$ : $\mathrm{Na}(+)$ entry and modulation of $\mathrm{Na}(+) / \mathrm{Ca}(2+)$ exchange as a key mechanism of TRPC signaling. Pflugers Arch 451: 99-104, 2005.

10. Pedersen SF, Owsianik G and Nilius B: TRP channels: An overview. Cell Calcium 38: 233-252, 2005.

11. Hogan PG and Rao A: Store-operated calcium entry: Mechanisms and modulation. Biochem Biophys Res Commun 460: 40-49, 2015.

12. Pagiatakis C, Gordon JW, Ehyai S and McDermott JC: A novel RhoA/ROCK-CPI-17-MEF2C signaling pathway regulates vascular smooth muscle cell gene expression. J Biol Chem 287: 8361-8370, 2012.

13. Eto M, Kirkbride JA, Chugh R, Karikari NK and Kim JI: Nuclear localization of CPI-17, a protein phosphatase-1 inhibitor protein, affects histone $\mathrm{H} 3$ phosphorylation and corresponds to proliferation of cancer and smooth muscle cells. Biochem Biophys Res Commun 434: 137-142, 2013.

14. Sasaki N and Sutoh K: Structure-function relationship of myosin. Tanpakushitsu Kakusan Koso 46 (11 Suppl): S1740-S1749, 2001 (In Japanese)

15. Ozaki H, Yasuda K, Kim YS, Egawa M, Kanzaki H, Nakazawa H, Hori M, Seto M and Karaki H: Possible role of the protein kinase C/CPI-17 pathway in the augmented contraction of human myometrium after gestation. Br J Pharmacol 140: 1303-1312, 2003.

16. Li W, Lv J, Wu J, Zhou X, Jiang L, Zhu X, Tu Q, Tang J, Liu Y, He A, et al: Maternal high-salt diet altered PKC/MLC20 pathway and increased ANG II receptor-mediated vasoconstriction in adult male rat offspring. Mol Nutr Food Res 60: 1684-1694, 2016.

17. Uneyama $\mathrm{H}$ and Takeuchi $\mathrm{K}$ : New therapeutic strategy for amino acid medicine: Preface. J Pharmacol Sci 118: 129-130, 2012.

18. Struck AW, Thompson ML, Wong LS and Micklefield J: S-adenosyl-methionine-dependent methyltransferases: highly versatile enzymes in biocatalysis, biosynthesis and other biotechnological applications. Chembiochem 13: 2642-2655, 2012.

19. Frezza M, Centini G, Cammareri G, Le Grazie C and Di Padova C: S-adenosylmethionine for the treatment of intrahepatic cholestasis of pregnancy. Results of a controlled clinical trial. Hepatogastroenterology 37 (Suppl 2): S122-S125, 1990.

20. De Silva V, El-Metwally A, Ernst E, Lewith G and Macfarlane GJ; Arthritis Research UK Working Group on Complementary and Alternative Medicines: Evidence for the efficacy of complementary and alternative medicines in the management of osteoarthritis: A systematic review. Rheumatology (Oxford) 50: 911-920, 2011.

21. Polli E, Cortellaro M, Parrini L, Tessari L and Chériè Lignière G: Pharmacological and clinical aspects of S-adenosylmethionine (SAMe) in primary degenerative arthropathy (osteoarthrosis). Minerva Med 66: 4443-4459, 1975 (In Italian).

22. Galizia I, Oldani L, Macritchie K, Amari E, Dougall D, Jones TN, Lam RW, Massei GJ, Yatham LN and Young AH: S-adenosyl methionine (SAMe) for depression in adults. Cochrane Database Syst Rev 10: CD011286, 2016.

23. Zhao Y, Li JS, Guo MZ, Feng BS and Zhang JP: Inhibitory effect of S-adenosylmethionine on the growth of human gastric cancer cells in vivo and in vitro. Chin J Cancer 29: 752-760, 2010.

24. Livak KJ and Schmittgen TD: Analysis of relative gene expression data using real-time quantitative PCR and the 2(-Delta Delta C(T)) method. Methods 25: 402-408, 2001.

25. Agrawal V and Hirsch E: Intrauterine infection and preterm labor. Semin Fetal Neonatal Med 17: 12-19, 2012. 
26. Bondarenko KM and Bondarenko VM: Bacterial lipopolsaccharides in pathogenesis of gynecological diseases and obstetric complications. Zh Mikrobiol Epidemiol Immunobiol: 80-86, 2014 (In Russian).

27. Song Z, Chen T, Deaciuc IV, Uriarte S, Hill D, Barve S and McClain CJ: Modulation of endotoxin stimulated interleukin- 6 production in monocytes and Kupffer cells by S-adenosylmethionine (SAMe). Cytokine 28: 214-223, 2004.

28. Song Z, Barve S, Chen T, Nelson W, Uriarte S, Hill D and McClain C: S-adenosylmethionine (AdoMet) modulates endotoxin stimulated interleukin-10 production in monocytes. Am J Physiol Gastrointest Liver Physiol 284: G949-G955, 2003.

29. Hevia H, Varela-Rey M, Corrales FJ, Berasain C, Martínez-Chantar ML, Latasa MU, Lu SC, Mato JM, García-Trevijano ER and Avila MA: 5'-methylthioadenosine modulates the inflammatory response to endotoxin in mice and in rat hepatocytes. Hepatology 39: 1088-1098, 2004.

30. Song Z, Zhou Z, Song M, Uriarte S, Chen T, Deaciuc I and McClain CJ: Alcohol-induced S-adenosylhomocysteine accumulation in the liver sensitizes to TNF hepatotoxicity: Possible involvement of mitochondrial S-adenosylmethionine transport. Biochem Pharmacol 74: 521-531, 2007.

31. Tang $Y$, Chu $H$, Cao G, Du $X$, Min $X$ and Wan $C$ : $\mathrm{S}$-Adenosylmethionine attenuates bile duct early warm ischemia reperfusion injury after rat liver transplantation. Mol Immunol 95: 83-90, 2018.

32. Zurabishvili S, Mamamtavrishvili I, Apridonidze K and Shanidze L: Role of intracellular infections in premature childbirth. Georgian Med News 126: 58-60, 2005.

33. Papiernik E: Premature childbirth and its prevention. Arch Fr Pediatr 34: 488-491, 1977 (In French).

34. Penev I: Etiopathogenesis of premature childbirth. Akush Ginekol (Sofiia) 23: 193-200, 1984 (In Bulgarian).

35. Renthal NE, Williams KC, Montalbano AP, Chen CC, Gao L and Mendelson CR: Molecular regulation of parturition: A myometrial perspective. Cold Spring Harb Perspect Med 5: pii: a023069, 2015.

36. Sanborn BM: Hormones and calcium: Mechanisms controlling uterine smooth muscle contractile activity. The litchfield lecture. Exp Physiol 86: 223-237, 2001.

37. Mark MD, Schwitalla JC, Groemmke M and Herlitze S: Keeping our calcium in balance to maintain our balance. Biochem Biophys Res Commun 483: 1040-1050, 2017

38. Blanks AM, Zhao ZH, Shmygol A, Bru-Mercier G, Astle S and Thornton S: Characterization of the molecular and electrophysiological properties of the T-type calcium channel in human myometrium. J Physiol 581: 915-926, 2007.
39. Collins PL, Moore JJ, Lundgren DW, Choobineh E, Chang SM and Chang AS: Gestational changes in uterine L-type calcium channel function and expression in guinea pig. Biol Reprod 63: 1262-1270, 2000

40. Zhang R, Luo H, Wang S, Chen W, Chen Z, Wang HW, Chen Y, Yang J, Zhang X, Wu W, et al: MicroRNA-377 inhibited proliferation and invasion of human glioblastoma cells by directly targeting specificity protein 1 . Neuro Oncol 16: 1510-1522, 2014.

41. Shi J, Miralles F, Birnbaumer L, Large WA and Albert AP: Store depletion induces Gaq-mediated PLC $\beta 1$ activity to stimulate TRPC1 channels in vascular smooth muscle cells. FASEB J 30: 702-715, 2016.

42. Ho JC and Lee CH: TRP channels in skin: From physiological implications to clinical significances. Biophysics (Nagoya-shi) 11: 17-24, 2015.

43. Zeng C, Tian F and Xiao B: TRPC channels: Prominent candidates of underlying mechanism in neuropsychiatric diseases. Mol Neurobiol 53: 631-647, 2016.

44. Kawarabayashi Y, Hai L, Honda A, Horiuchi S, Tsujioka H, Ichikawa $\mathbf{J}$ and Inoue R: Critical role of TRPC1-mediated $\mathrm{Ca}^{2+}$ entry in decidualization of human endometrial stromal cells. Mol Endocrinol 26: 846-858, 2012.

45. Ku CY, Babich L, Word RA, Zhong M, Ulloa A, Monga M and Sanborn BM: Expression of transient receptor channel proteins in human fundal myometrium in pregnancy. J Soc Gynecol Investig 13: 217-225, 2006.

46. Zheng D, Zhang L, Na Q, Liu S, Zhuang Y, Lv Y and Liu C: Enhanced expression of transient receptor potential channel 3 in uterine smooth muscle tissues of lipopolysaccharide-induced preterm delivery mice. Iran J Basic Med Sci 19: 567-572, 2016.

47. Su W, Xie Z, Liu S, Calderon LE, Guo Z and Gong MC: Smooth muscle-selective CPI-17 expression increases vascular smooth muscle contraction and blood pressure. Am J Physiol Heart Circ Physiol 305: H104-H113, 2013.

48. Swärd K, Mita M, Wilson DP, Deng JT, Susnjar M and Walsh MP: The role of RhoA and Rho-associated kinase in vascular smooth muscle contraction. Curr Hypertens Rep 5: 66-72, 2003.

This work is licensed under a Creative Commons

Attribution-NonCommercial-NoDerivatives 4.0 International (CC BY-NC-ND 4.0) License. 